\title{
Granularity-induced gapless superconductivity in NbN films: evidence of thermal phase fluctuations
}

\author{
G. Lamura ${ }^{1, \dagger}$, J.-C. Villégier ${ }^{2}$, A. Gauzzi ${ }^{3, *}$, J. Le Cochec ${ }^{1, \S}$, \\ J.-Y. Laval ${ }^{1}$, B. Plaçais ${ }^{4}$, N. Hadacek ${ }^{2}$, and J. Bok ${ }^{1}$ \\ 1 LPS-ESPCI, 10, rue Vauquelin, 75231 Paris, France. \\ ${ }^{2}$ CEA-Grenoble, SPSMS-LCP, 17, rue des Martyrs, 38054 Grenoble, France. \\ ${ }^{3}$ MASPEC-CNR, Area delle Scienze, 43010 Parma, Italy. \\ ${ }^{4}$ LPMC-ENS, UMR 8551, 24, rue Lhomond, 75231 Paris, France.
}

(Dated: February 7, 2020)

\begin{abstract}
Using a single coil mutual inductance technique, we measure the low temperature dependence of the magnetic penetration depth in superconducting NbN films prepared with similar critical temperatures $\approx 16 \mathrm{~K}$ but with different microstructures. Only (100) epitaxial and weakly granular (100) textured films display the characteristic exponential dependence of conventional BCS $s$-wave superconductors. More granular (111) textured films exhibit a linear dependence, indicating a gapless state in spite of the $s$-wave gap. This result is quantitatively explained by a model of thermal phase fluctuations favored by the granular structure.
\end{abstract}

PACS numbers: 74.25.Nf, 74.40.+k, 74.50.+r, 74.80.Bj 
Distinctive property of simple superconducting materials like metals and alloys is the absence of thermally activated electronic excitations at low temperature $T \ll T_{c}$, where $T_{c}$ is the critical temperature. This property is consistent with a model of superconducting gap $\Delta_{\mathbf{k}}$ with $s$-wave symmetry, as predicted by the BCS theory. In contrast, in complex materials like heavy fermions [1], cuprates [2] or rhutenates [3], thermal excitations of arbitrarily small energies are experimentally observed. This gapless property has been commonly explained by an excitation spectrum with nodal points or lines, characteristic of $\Delta_{\mathbf{k}}$ with $p$ - or $d$-symmetry respectively. According to theoretical considerations, non- $s$ symmetries are favored by nonphonon mediated pairing mechanisms like antiferromagnetic spin fluctuations [4]. However, experimental and theoretical studies show that a gapless state can be induced in $s$-wave superconductors by residual interactions coexisting with the pairing interaction, such as magnetic impurities [5], spin density waves [6], proximity effects [7, 8] or phase fluctuations of the order parameter [9, 10, 11]. In conclusion, a non- $s$ symmetry implies a gapless state, but the opposite does not hold.

In this work we investigate whether the above predictions of gapless state apply to the $s$-wave superconductor NbN. By measuring the variation of the magnetic penetration depth $\lambda(T)-\lambda(0) \equiv \delta \lambda(T)$ at $T \geq 1.5 \mathrm{~K}$, we study the low energy excitations on films with different microstructures obtained by varying growth conditions. $\mathrm{NbN}$ is a model system for our purpose taking into account the following: $i$ ) the simple cubic structure with fcc lattice [12]; ii) the conventional BCS $s$-wave superconducting properties [13]; iii) the relatively high critical temperature $T_{c} \approx 16 \mathrm{~K}$ enables to study the low energy excitations in a wider temperature region; $i v$ ) various materials preparation routes have been developed and optimized, especially in view of electronics applications 14, 15, 16.

For this work we have selected six NbN films prepared by DC magnetron sputtering with similar $T_{c}$ close to the bulk value $\approx 16 \mathrm{~K}$ but with different degrees of granularity obtained by varying growth conditions. These conditions are reported in Table I (see [15, 16] for more details): growth temperatures are either room temperature or $620^{\circ} \mathrm{C}$; substrates are $(1 \overline{1} 02)$ $\mathrm{Al}_{2} \mathrm{O}_{3}$ or (100) $\mathrm{MgO}$ single crystals or (1102) $\mathrm{Al}_{2} \mathrm{O}_{3}$ single crystals with a $12 \mathrm{~nm}$ thick (100) $\mathrm{MgO}$ buffer layer; NbN thicknesses range from 10 to $1400 \mathrm{~nm}$. Three different types of microstructure are reproducibly obtained: i) (100) epitaxial; ii) highly (100) textured; iii) (111) textured. Using (100) MgO buffer layers, a high degree of (100) texture is obtained only in sufficiently thin $(d \lesssim 150 \mathrm{~nm}) \mathrm{NbN}$ films [16]. 
The film microstructure is characterized by X-ray diffraction in the Bragg-Brentano geometry, X-ray pole figure measurements and transmission electron and atomic force microscopies (TEM, AFM). X-ray rocking curve analysis indicates that samples A1043 and A1013 are epitaxial with FWHM of the (420) Bragg refelection less than $1^{\circ}$. The former sample is thin $(10 \mathrm{~nm})$, whereas the latter is thick $(950 \mathrm{~nm})$. The film on the $\mathrm{MgO}$ buffer layer (A1044) is thin $(10 \mathrm{~nm})$ and highly (100) textured. The remaining three films on sapphire (A1057, A1060 and A1063) are all thick (280-1400 nm) and display predominant (111) texture. The different degrees of granularity in these films are confirmed by AFM and TEM. For example, in sample A1043, we find an atomically flat surface [17] and a crystal structure with apparently no grain boundaries (see fig. 1a), which indicates that this film is monocrystalline. A granular structure is evident in the (111) textured sample A1060 from the TEM cross-sectional micrograph of fig. 1b. We note a columnar structure with grains of size 15-35 nm and well-defined grain boundaries, as previously reported for films grown under the same conditions [15.

The microstructural properties of the six films are correlated with DC resistivity $\varrho$ and DC transport critical current density $j_{c}$ measurements. The results of these measurements are summarized in Table I. In all films, $T_{c}$ is close to the bulk value $\approx 16 \mathrm{~K}$, which indicates that the microstructure does not appreciably perturb the superconducting phase. Smaller residual resistivities $\varrho(20 \mathrm{~K}) \approx 50 \mu \Omega \mathrm{cm}$ and larger resistivity ratios $\varrho(300 \mathrm{~K}) / \varrho(20 \mathrm{~K}) \approx 1.2$ are found in the epitaxial films. The highest values of $j_{c}(4.2 \mathrm{~K}) \gtrsim 3 \mathrm{MA} / \mathrm{cm}^{2}$ are found in the epitaxial or thin (100) textured samples, while the three thick (111) textured ones exhibit values $\lesssim 1 \mathrm{MA} / \mathrm{cm}^{2}$ that decrease with increasing $\mathrm{NbN}$ thickness. In agreement with earlier studies [15] and with our TEM results, we conclude the following: $i$ ) the epitaxial samples have higher crystalline quality; ii) in the (111) textured samples, the degree of granularity - defined as the degree of grain decoupling - increases with increasing thickness.

To correlate the above structural and transport properties with the low energy excitations, we have carried out $\delta \lambda(T)$ measurements in the range $1.5 \mathrm{~K}-T_{c}$ using a single-coil mutual inductance technique described elsewhere [18]. Typical frequency and magnitude of the e.m. field perpendicular to the film surface are $2-4 \mathrm{MHz}$ and $<0.1 \mathrm{mT}$ respectively. Care has been taken to always operate in the linear response regime, i.e. below the Josephson critical field $B_{c 1}$, monitored by varying the applied magnetic field. Note that we measure an effective magnetic penetration depth that, in the dirty limit, differs from the London penetration 
depth by a correction factor of the order of unity [19]. This limit is appropriate for our films, for which we estimate a mean free path $\approx 1 \mathrm{~nm}$ and a coherence length $\xi \approx 3-5 \mathrm{~nm}$ [15, [17]. As a good approximation, the above correction factor is temperature independent in the limited temperature range of our measurements. Therefore the temperature dependence of the variations $\delta \lambda(T)$ is not affected by dirty limit effects.

In figs. 2a-b, we report the experimental $\delta \lambda(T)$ curves obtained. Only the epitaxial and thin (100) textured films display the exponential behavior $\delta \lambda \sim \exp \left(-\Delta / k_{B} T\right)$, characteristic of BCS $s$-wave superconductors [20] (see fig. 2a). All of the three thick (111) textured samples exhibit a linear behavior which can not be approximated by any power law (see fig. $2 \mathrm{~b}$ ); the range of linearity is limited below $\approx 2.7 \mathrm{~K}$ in the thinner film A1057 and extends up to $\approx 4 \mathrm{~K}$ in the thicker films A1060 and A1063. A BCS $s$-wave fit perfectly accounts for the experimental data of the three samples of fig. 2a. $\lambda(0)$ is the only free parameter of this fit, whereas the ratio $2 \Delta(0) / k_{B} T_{c}$ is kept fixed at 4.6, taken from tunneling data at $1.5 \mathrm{~K}$ on sample A1043. The fitted $\lambda(0)$ values are in the range $200-400 \mathrm{~nm}$, in agreement with previous reports [13, [16]. A BCS fit is satisfactory also for the thinner (111) textured film A1057 of fig. 2b; indeed, the behavior of this sample is intermediate between exponential and linear. For the remaining two samples A1060 and A1063, no BCS fit is possible for any plausible set of parameters.

These results give evidence of a gapless superconducting state in the (111) textured films. We recall the mechanisms that may induce a gapless state in a conventional BCS $s$-wave superconductor: i) magnetic impurities; ii) proximity effects; iii) phase fluctuations of the order parameter. In our case, the first two possibilities are excluded: $i$ ) the purity of the $\mathrm{Nb}$ target used is $99.995 \%$ and traces of magnetic impurities are below $10 \mathrm{ppm}$. We conclude that the amount of these impurities in the films is negligible. In any case, the gapless samples contain no more impurities than the others, since all of them are prepared with the same $\mathrm{Nb}$ target; ii) a previous TEM and tunneling study [15] indicates that the intergranular phases are insulating, thus no proximity effects are expected. The third picture remains plausible: as suggested earlier [9, 10, 11, 21, 22, 23], both quantum and thermal phase fluctuations can be relevant in granular superconductors with short coherence length $\xi$ like our $\mathrm{NbN}$ films.

To verify the validity of the above picture in our case, we consider an array of superconducting grains with intergranular Josephson coupling energy $E_{J}$ and charging energy $U$, as first proposed by Abeles [24], where $U$ is associated with the excess of Cooper pairs $n$ 
in each grain. $U$ and $E_{J}$ are related to the intergranular capacitance $C$ and the critical current $I_{c}$ respectively, according to the relations $U=2 e^{2} / C$ and $E_{J}=\hbar I_{c} / 2 e$. Following previous work [22, 25], we assume the array to form a square lattice of constant $a$ equal to the average grain size. The resulting Hamiltonian is:

$$
H=\frac{U}{2} \sum_{i} n_{i}{ }^{2}+E_{J} \sum_{i \neq j}\left[1-\cos \left(\vartheta_{i}-\vartheta_{j}\right)\right]
$$

where $\vartheta_{i}$ is the phase of the order parameter in the $i$-th grain and $n_{i}=-2 i \partial / \partial \vartheta_{i}$ is its conjugate operator.

Eq. (1) describes both quantum and classical phase fluctuations. Two arguments suggest a classical description of our results in the range $T \geq 1.5 \mathrm{~K}$ of our experiment. $i$ ) According to theoretical studies [9, 23] supported by numerical calculations [10], quantum fluctuations lead to a reduction of the renormalized superfluid density, implying a progressive decrease of $\partial \lambda / \partial T$ as $T \rightarrow 0 \mathrm{~K}$. This effect is not observed in the curves of fig. $2 \mathrm{~b}$ which remain linear above $1.5 \mathrm{~K}$. ii) In our (111) textured films, we estimate $U \ll E_{J}$, which corresponds to a purely classical regime [9, 22, 23]. Indeed, typical figures are: $a \approx 20 \mathrm{~nm}, \lambda(0) \approx 300 \mathrm{~nm}$, $j_{c} \approx 0.5-1 \mathrm{MA} / \mathrm{cm}^{2}$, thickness and relative dielectric constant of the intergranular regions respectively $t \lesssim 2 \mathrm{~nm}$ and $\epsilon_{r} \gtrsim 2$. Thus, we obtain $E_{J} \approx 50-100 \mathrm{meV}$ and $U \lesssim 10 \mu \mathrm{eV}$.

In the classical regime, the fluctuation strength is determined by the ratio $E_{J} / k_{B} T_{c}$, where $T_{c}$ is the mean field critical temperature. Using the above estimate, we find $E_{J} \gg k_{B} T_{c}$ in our granular films. Thus, according to previous studies [22, 25, we expect small fluctuations which do not inhibit long range phase order at any temperature. Indeed, we do not observe any appreciable decrease of $T_{c}$ induced by granularity $\left(T_{c}\right.$ is even slightly larger in the (111) textured films). These fluctuations coexist with the usual quasiparticle excitations predicted for BCS $s$-wave superconductors. Both excitations lead to a progressive increase of $\lambda$ as temperature increases. In the Gaussian approximation, valid at low temperatures $T \ll E_{J} / k_{B}$ and in the absence of dissipation, only long wavelength longitudinal fluctuations of arbitrarily small energy are created (Goldstone modes). This produces a linear increase $\delta \lambda \sim T$ 19, 10, 11] experimentally observed in fig. 2b. On the other hand, the creation of quasiparticles requires a finite thermal energy $\gtrsim \Delta$, which leads to the exponential dependence of fig. $2 \mathrm{a}$.

We now analyze in quantitative form the experimental curves of fig. $2 \mathrm{~b}$ within the framework of the above picture. Using Table I, we estimate first the fluctuation contribution and 
then the temperature at which the quasiparticle contribution becomes significant. Following previous calculations [9, 10], in the Gaussian regime, our lattice model with $U=0$ leads to:

$$
\frac{\partial \lambda}{\partial T}=\frac{e k_{B}}{\hbar} \frac{1}{j_{c} a}
$$

where $I_{c} \approx j_{c} a \lambda$ at $0 \mathrm{~K}$ and we neglect the weak temperature dependence of this product at low temperature. In Table I we report the values of $\partial \lambda / \partial T$ estimated using eq. (2) and compare them with the experimental values. Taking into account the uncertainty in the estimate of $a$, we notice a good agreement. Interestingly, the trend of $j_{c}$ in the (111) textured films follows the trend of $\partial \lambda / \partial T$, as predicted by eq. (2): the thicker the film, the smaller $j_{c}$ and the larger the slope $\partial \lambda / \partial T$. In the films of fig. 2a, exhibiting a BCS $s$-wave behavior, $j_{c}$ is larger. Thus, the fluctuation effect vanishes and only the quasiparticle effect is observed. To estimate the temperature at which the two effects become comparable, we separately evaluate their contribution to $\partial \lambda / \partial T$ using Table I and the BCS fit of fig. $2 \mathrm{~b}$. We find that the linear increase of $\lambda$ produced by thermal phase fluctuations is expected to dominate at $T \lesssim 4 \mathrm{~K}$. This is in agreement with the point of departure from linearity in samples A1060 and A1063. As discussed before, in sample A1057 a gapless behavior is visible but does not dominate yet above $1.5 \mathrm{~K}$, since a BCS fit is still satisfactory.

In conclusion, we have reported for the first time experimental evidence of gapless state in a conventional BCS $s$-wave superconductor, NbN. Evidence is found in granular films exhibiting a clear linear dependence of $\lambda$ in the range 1.5-4 K. Epitaxial or less granular films exhibit the conventional dependence of $s$-wave superconductors and no trace of gapless state is found. A simple model of granularity-induced thermal phase fluctuations of the order parameter (Goldstone modes) quantitatively accounts for the above linear dependence. No signature of quantum fluctuations is observed above $1.5 \mathrm{~K}$, as expected taking into account the relatively large grains of our films.

Our evidence of granularity-induced gapless superconductivity in $\mathrm{NbN}$ raises the question whether this phenomenon is observable in other granular superconductors. In cuprates, which display gapless properties in agreement with a $d$-wave symmetry model, it should be investigated if these properties could be - at least partially - explained by intrinsic granular properties associated with chemical or electronic inhomogeneities in the coherence length scale $\lesssim 3 \mathrm{~nm}$. In any case, our results suggest that the only reliable methods for testing 
non- $s$ symmetries are those based on phase-sensitive measurements.

\section{Acknowledgments}

We acknowledge A. Andreone, A. Barone, B. K. Chakraverty and G. Deutscher for stimulating discussions and A. Dubon for assistance in the TEM work.

*Corresponding author. E-mail address: gauzzi@maspec.bo.cnr.it

${ }^{\dagger}$ Present address is: INFM and Dipartimento di Scienze Fisiche, Università di Napoli Federico II, 80125 Napoli, Italy.

§Present address is: Laboratoire Pierre Süe, DRECAM, CEA-Saclay, 91191 Gif sur Yvette, France. 
[1] F. Gross, B. S. Chandrasekhar, D. Einzel, K. Andres, P. J. Hirschfeld, H. R. Ott, J. Beuers, Z. Fisk, and J. L. Smith, Z. Phys. B: Condens. Matter 64, 175 (1986).

[2] W.N. Hardy, D.A. Bonn, D.C. Morgan, R. Liang, and K. Zhang, Phys. Rev. Lett. 70, 3999 (1993).

[3] I. Bonalde, B.D. Yanoff, M.B. Salamon, D.J. Van Harlingen, E.M.E. Chia, Z.Q. Mao and Y. Maeno, Phys. Rev. Lett. 85, 4775 (2000).

[4] See, for example, P. Monthoux, A.V. Balatsky, and D. Pines, Phys. Rev. Lett. 72, 1874 (1994) and references therein.

[5] A.A. Abrikosov and L.P. Gor'kov, Zh. Eksp. \& Teor. Fiz. 39, 178 (1960); P. Fulde, R. A. Ferrel, Phys. Rev. 135, A550 (1964); A.I. Larkin, Y.N. Ovchinnikov, JETP 20, 762 (1965).

[6] A.W. Overhauser and L.L. Daemen, Phys. Rev. Lett. 61, 1885 (1988).

[7] J.H. Claassen, J.E. Evetts, R.E. Somekh, and Z.H. Barber, Phys. Rev. B 44, 9605 (1991).

[8] M.S. Pambianchi, L. Chen and S.M. Anlage, Phys. Rev. B 54, 3508 (1996).

[9] M.W. Coffey, Physica C 235-240, 1961 (1995).

[10] E. Roddick and D. Stroud, Phys. Rev. Lett. 74, 1430 (1995).

[11] V.J. Emery and S.A. Kivelson, Phys. Rev. Lett. 74, 3253 (1995).

[12] K. Becker and F. Ebert, Z. für Physik 31, 268 (1925).

[13] M.S. Pambianchi, S.M. Anlage, E.S. Hellman, E.H. Hartford Jr., M. Bruns and S.Y.Lee, Appl. Phys. Lett. 64, 244 (1994); B. Komiyama, Z. Wang and M. Tonouchi, Appl. Phys. Lett. 68, $562(1996)$.

[14] See, for example, D.D. Bacon, A.T. English, S. Nakahara, F.G. Peters, H. Schreiber, W.R. Sinclair, and R.B. van Dover, J. Appl. Phys. 54, 6509 (1983).

[15] J.-C. Villégier, L. Vieux-Rochaz, M. Goniche, P. Renard, M. Vabre, IEEE Trans Magn. 21, 498 (1985); R. Chicault and J.-C. Villégier, Phys. Rev. B. 36, 5215 (1987).

[16] J.-C. Villégier, B. Delaët, V. Larrey, P. Febvre, J.W. Tao and G. Angenieux, Physica C 326-327, 133 (1999).

[17] G. Lamura, PhD Thesis, Université Paris VI (2001).

[18] A. Gauzzi, J. Le Cochec, G. Lamura, B.J. Jönsson, V.A. Gasparov, F.R. Ladan, B. Plaçais, P.-A. Probst, D. Pavuna and J. Bok, Rev. Sci. Instr. 71, 2147 (2000). 
[19] M. Tinkham in Introduction to Superconductivity (McGraw-Hill, Singapore, 1996), p. 97.

[20] J. Bardeen, L. N. Cooper, and J. R. Schrieffer, Phys. Rev. 108, 1175 (1957).

[21] G. Deutscher, Y. Imry and L. Gunther, Phys. Rev. B, 10, 4598 (1974).

[22] E. Šmánek, Solid state Comm. 31, 419 (1979).

[23] B.K. Chakraverty, Physica C 341-348, 75 (2000) and references therein.

[24] B. Abeles, Phys. Rev. B 15, 2828 (1977).

[25] W.L. McLean and M.J. Stephen, Phys. Rev. B 19, 5925 (1979). 
FIG. 1: a). Cross-sectional TEM image of the (100) epitaxial sample A1043. Note the monocrystalline structure evidenced by the contrast produced by the diagonal (110) planes. The notation "R" indicates the (1102) orientation of the sapphire substrate. b). The same as in a) for the thick (111) textured A1060 sample. Note the granular structure made of columnar grains.

FIG. 2: a). Temperature dependence of the magnetic penetration depth $\lambda$ measured in the films with conventional BCS s-wave behavior: A1013 (full circles), A1043 (squares) and A1044 (open circles). b). The same as in a) for the films with gapless behavior: A1057 (lozenges), A1060 (full triangles) and A1063 (open triangles). The BCS-like curve of sample A1044 (circles) of a) and the corresponding BCS $s$-wave fit (solid line) are also shown for comparison.

TABLE I: Summary of sample properties and comparison between experimental values of $\partial \lambda / \partial T$ and theoretical ones obtained using eq. (2). For the samples following the BCS thermally activated behavior, $\lambda(0)$ is obtained using a BCS $s$-wave fit, as discussed in the text.

\begin{tabular}{|l|c|c|c|c|c|c|}
\hline sample & $\mathbf{A 1 0 4 3}$ & $\mathbf{A 1 0 1 3}$ & $\mathbf{A 1 0 4 4}$ & $\mathbf{A 1 0 5 7}$ & $\mathbf{A 1 0 6 0}$ & $\mathbf{A 1 0 6 3}$ \\
\hline \hline NbN thickness $[\mathrm{nm}]$ & 10 & 950 & 10 & 280 & 680 & 1400 \\
substrate & $\mathrm{Al}_{2} \mathrm{O}_{3}$ & $\mathrm{MgO}$ & $\mathrm{Al}_{2} \mathrm{O}_{3} / \mathrm{MgO}$ & $\mathrm{Al}_{2} \mathrm{O}_{3}$ & $\mathrm{Al}_{2} \mathrm{O}_{3}$ & $\mathrm{Al}_{2} \mathrm{O}_{3}$ \\
substrate orient. & $(1 \overline{1} 02)$ & $(100)$ & $(1 \overline{1} 02) /(100)$ & $(1 \overline{1} 02)$ & $(1 \overline{1} 02)$ & $(1 \overline{102})$ \\
growth temp. $\left[{ }^{\circ} \mathrm{C}\right]$ & 620 & room temp. & 620 & room temp. & room temp. & room temp. \\
microstructure & epitaxial & epitaxial & highly textured & textured & textured & textured \\
NbN orientation & $(100)$ & $(100)$ & $(100)$ & $(111)$ & $(111)$ & $(111)$ \\
$T_{c}[\mathrm{~K}]$ & $14.2 \pm 0.1$ & $15.5 \pm 0.09$ & $14.4 \pm 0.1$ & $13.4 \pm 0.1$ & $16.1 \pm 0.1$ & $16.3 \pm 0.1$ \\
$j_{c}\left[\mathrm{MA} / \mathrm{cm}{ }^{2}\right]$ & $3.0 \pm 0.7$ & - & $9.0 \pm 0.3$ & $1.3 \pm 0.7$ & $1.1 \pm 0.3$ & $0.64 \pm 0.04$ \\
$\varrho(20 \mathrm{~K})[\mu \Omega \mathrm{cm}]$ & 53 & 50 & 147 & 448 & 88 & 67 \\
$\varrho(300 \mathrm{~K}) / \varrho(20 \mathrm{~K})$ & 1.2 & 1.2 & 0.9 & 0.7 & 0.9 & 1.0 \\
$\lambda(0)[\mathrm{nm}](\mathrm{BCS}$ fit $)$ & 240 & 260 & 270 & 470 & - & - \\
$\partial \lambda / \partial T$ exp. $[\AA / \mathrm{K}]$ & 0 & 0 & 0 & $2.1 \pm 0.5$ & $3.3 \pm 0.2$ & $5.6 \pm 0.5$ \\
$\partial \lambda / \partial T$ th. $[\AA / \mathrm{K}]$ & 0 & 0 & 0 & 1.6 & 2.2 & 3.3 \\
\hline
\end{tabular}


This figure "Lamura_Fig1a.jpg" is available in "jpg" format from: http://arxiv.org/ps/cond-mat/0201296v1 
This figure "Lamura_Fig1b.jpg" is available in "jpg" format from: http://arxiv.org/ps/cond-mat/0201296v1 


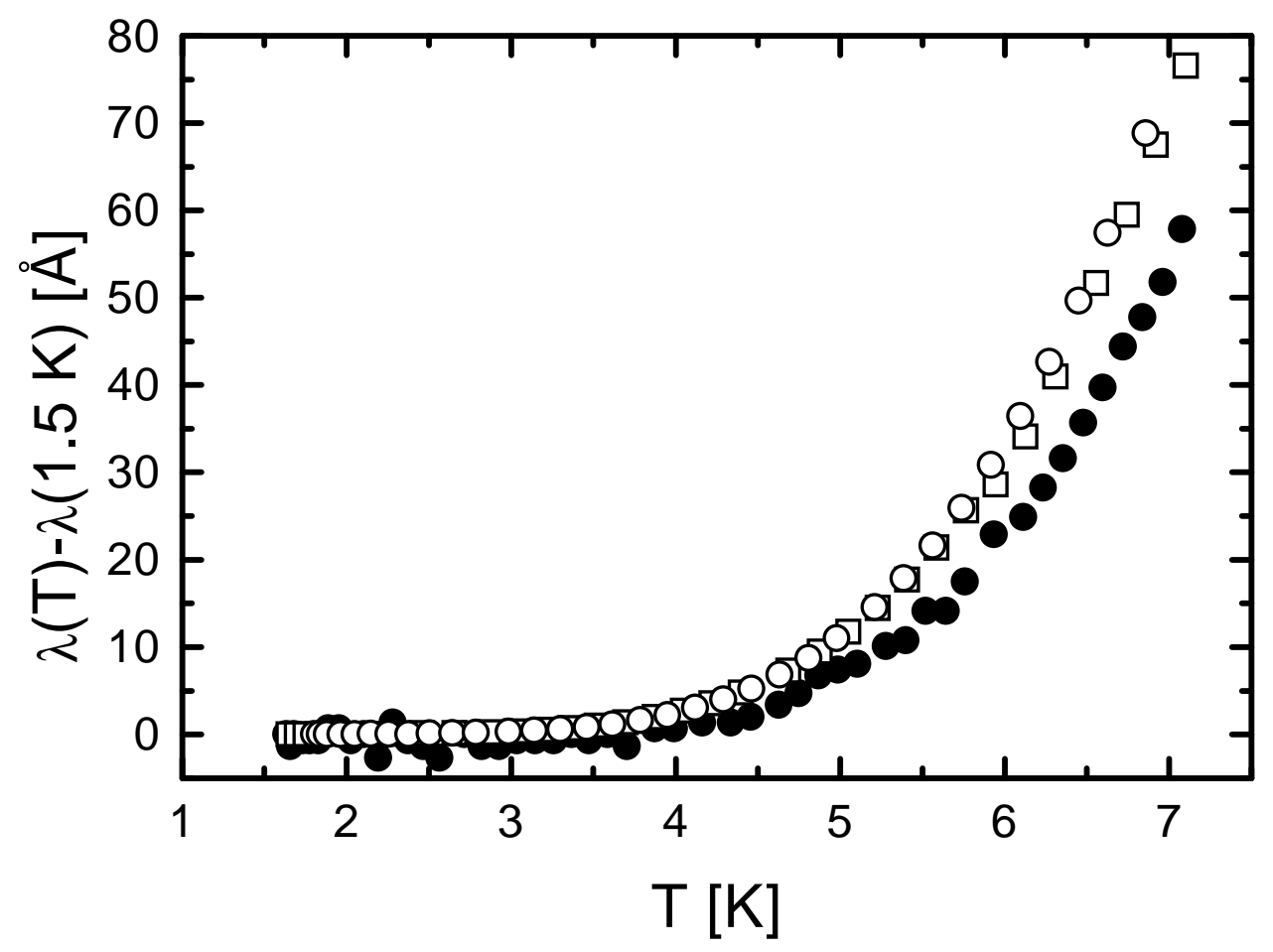




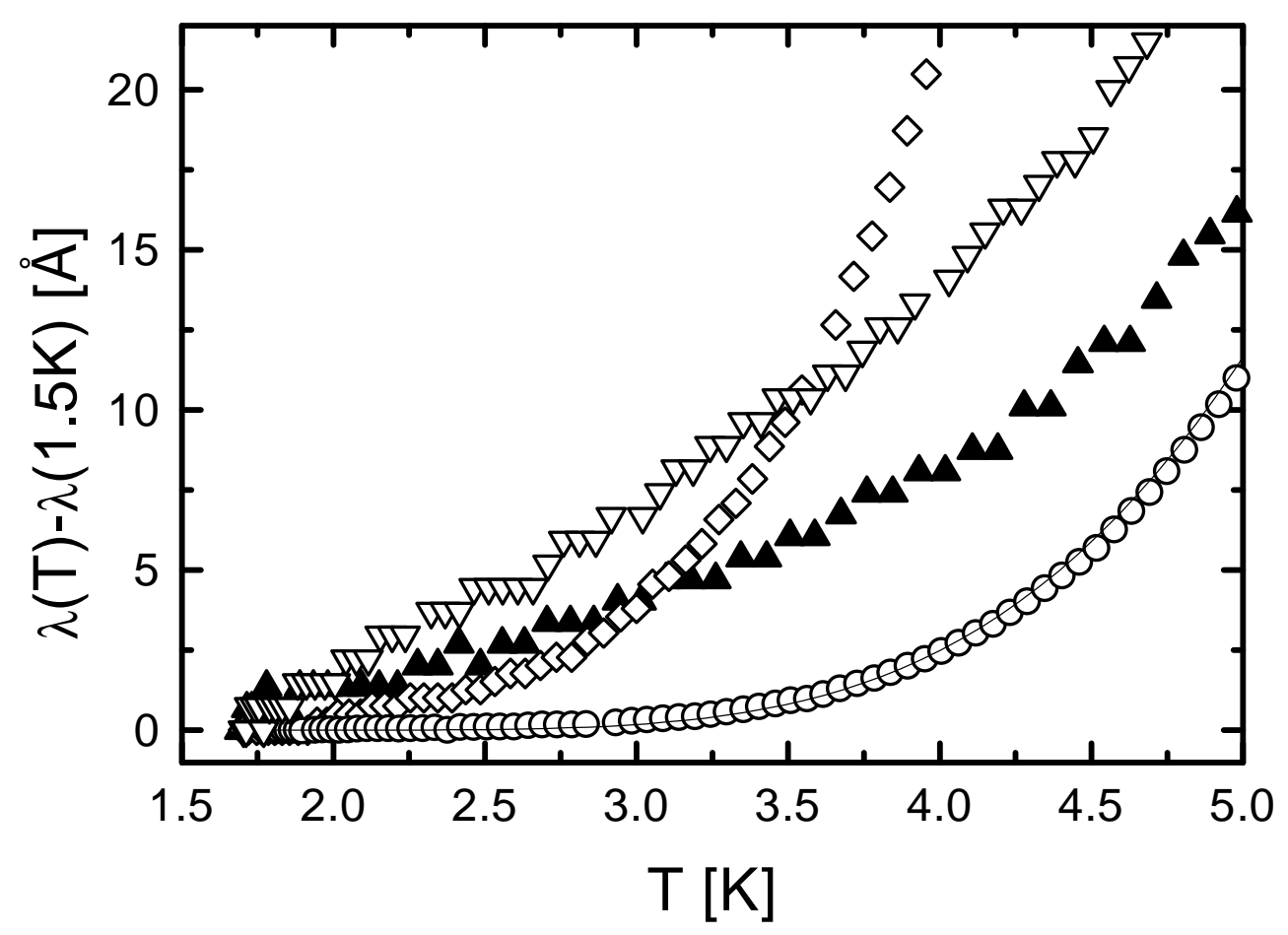

